\title{
Autopsy findings in 42 consecutive patients with idiopathic pulmonary fibrosis
}

\author{
C.E. Daniels*, E.S. Yi ${ }^{\#}$ and J.H. Ryu*
}

ABSTRACT: Idiopathic pulmonary fibrosis (IPF) is a relentlessly progressive lung disease that commonly results in respiratory failure and death. However, the cause of death in these patients has not previously been fully defined.

The current study reviews the clinical records and pathological findings of 42 consecutive patients with IPF who underwent a post mortem at the Mayo Clinic (Rochester, MN, USA) over a 9-yr period, from January 1996 to December 2004.

The median (range) age at post mortem for the patients was 74 (46-98 yrs) yrs, which included $25(60 \%)$ males. A total of $31(74 \%)$ patients died in the hospital. The immediate causes of death were reported as: respiratory $(64 \%)$, cardiovascular $(21 \%)$, or noncardiopulmonary $(14 \%)$. Acute exacerbation of IPF was the most common immediate cause of death (29\%). Pneumonia, aspiration and drug-induced lung disease were identified as other causes of respiratory death. Evidence of pulmonary hypertension was present in the post mortem of $19(45 \%)$ patients and was the immediate cause of death in two of these patients. The immediate cause of death was clinically unsuspected in five (12\%) patients and IPF was diagnosed post mortem in nine $(21 \%)$ patients.

The majority of patients with idiopathic pulmonary fibrosis who had undergone a post mortem were found to have died from respiratory causes. Acute exacerbation of idiopathic pulmonary fibrosis was found to be the most common cause of death whilst death from the gradual progression of idiopathic pulmonary fibrosis was found to be less common.

KEYWORDS: Interstitial lung disease, lung, lung fibrosis, pathology diopathic pulmonary fibrosis (IPF) is an interstitial lung disease of unknown cause correlating with a histopathological pattern of usual intersitial pneumonia (UIP). In most patients the fibrotic process proceeds until it results in end-stage honeycombing and respiratory failure; the median survival is $\sim 2-3$ yrs after diagnosis [1]. There is no effective medical therapy for patients with IPF [2]. Lung transplantation is an option for younger patients without significant extrapulmonary disorders.

It is generally assumed that mortality in IPF is the result of progressive fibrotic lung disease leading to respiratory insufficiency. However, relatively few studies exist to verify or refute this assumption. Conditions associated with IPF, such as pulmonary hypertension and more recently acute exacerbation of IPF, are now recognised as potential causes of mortality in IPF patients [3, 4]. Clinicians and investigators are targeting these associated conditions and complications in the hope of extending and improving the lives of patients with IPF. However, the pathological contribution of these processes, as well as the natural history of progressive fibrosis resulting in death, remains largely uncertain. In addition, the presentation and pathological findings of patients who are diagnosed with IPF post mortem have not been well defined.

More precise data pertaining to the cause of death in IPF patients could focus clinical and research efforts and may improve survival. In the current study, the results of 42 consecutive post mortems performed on patients with IPF were examined to determine the immediate cause of death and associated findings.

\section{MATERIALS AND METHODS}

A total of 42 patients with IPF who underwent a post mortem at the Mayo Clinic (Rochester, MN, USA) over a 9-yr period from January 1, 1996 to December 31, 2004 were identified by imaging studies and a computer-assisted search of medical and post mortem records. The diagnosis of IPF required post mortem evidence of (UIP) with no connective tissue disease or exposure to fibrogenic drugs or environmental agents having been identified. The current study was approved by
AFFILIATIONS

*Division of Pulmonary and Critical Care Medicine, and

\#Dept of Laboratory Medicine and Pathology, Mayo Clinic, Rochester, MN, USA.

CORRESPONDENCE

Division of Pulmonary and Critical

Gonda South 18

Mayo Clinic

200 1st St. SW

Rochester

MN 55905

USA

Fax: 15072664372

E-mail: daniels.craig@mayo.edu

Received:

December 282007

Accepted after revision:

January 292008

SUPPORT STATEMENT

The present research was funded by the Mayo Foundation (Rochester,

MN, USA) and the Robert N. Brewer Family Foundation (Herrin, IL, USA)

STATEMENT OF INTEREST

None declared.
C.E. Daniels

Care Medicine 
the Mayo Foundation institutional review board (Rochester, MN, USA).

All subjects underwent a complete post mortem examination with the exception of four patients who underwent a partial post mortem (heart and lung only in three patients, lung only in one patient). Cause of death was defined as irreversible organ dysfunction that either resulted directly in the patient's death or in the withdrawal of life support. The immediate cause of death was defined as the final disease or complication directly causing death [5]. The contributing cause of death was defined as another significant condition or disease that contributed to death, but was not the immediate cause of death [5]. Clinician's diagnosis regarding the cause(s) of death was derived from a post mortem request form submitted by clinicians or a review of medical records by the current study's investigators. The actual cause of death was assigned by consensus from two of the current study's authors (C.E. Daniels and J.H. Ryu) based on a review of all available data. Discordance between clinician's diagnosis and actual cause of death was assessed.

All patients met the diagnostic criteria for IPF as outlined in the American Thoracic Society and European Respiratory Society consensus statement [6]. Acute exacerbation of IPF was defined using the following criteria proposed by $\mathrm{KONDOH}$ et al. [7] and AKIRA et al. [8]. 1) Acute worsening of dyspnoea within 1 month of presentation. 2) New pulmonary infiltrates on a chest radiograph or computed tomography scan. 3) Deterioration in pulmonary function measurements or gas exchange. 4) Absence of an identifiable cause including infections or cardiovascular disease. Documentation of new infiltrates or precipitous decline in lung function was not required in patients without a prior diagnosis of IPF since baseline results were not available.

\section{RESULTS}

The median (range) age of the 42 subjects at post mortem was 74 (46-98) yrs and included $25(60 \%)$ males (table 1). Overall, the immediate causes of death were respiratory, cardiovascular and noncardiopulmonary failings in 64,21 and $14 \%$ of the patients, respectively.

A total of $11(24 \%)$ patients were not hospitalised at the time of death. Comfort-care treatment preceded death in four of these patients, six died from an acute cardiac cause and one died from head trauma related to a fall. The remaining 31 patients died in hospital.

\section{TABLE 1 Demographical data for the 42 patients with} idiopathic pulmonary fibrosis that underwent a post mortem

\begin{tabular}{lc} 
Characteristic & \\
\hline Males & $25(60)$ \\
Age yrs & $73 \pm 11.8,74(46-98)$ \\
Smoking & 0 \\
$\quad$ Current & $18(43)$ \\
Previous & $24(57)$ \\
None & \\
\hline & \\
Data are presented as $n(\%)$ or mean \pm SD, median (range).
\end{tabular}

\section{Respiratory causes}

IPF was the immediate cause of death in 17 (40\%) of the 42 patients studied. In 12 (71\%) of these patients' histopathological evidence of diffuse alveolar damage (DAD) superimposed on UIP was present in the post mortem. In these cases, DAD correlated with acute clinical deterioration, hypoxia and radiographical progression of bilateral lung infiltrates; now termed acute exacerbation of IPF [7, 8]. Acute worsening of dyspnoea occurred over a median (range) duration of 7 (1- 28) days prior to hospitalisation. The median (range) duration of hospitalisation until death was 9 (1-43) days. Three of these patients underwent surgical lung biopsy during their final hospitalisation, which revealed DAD superimposed on UIP in all three patients. Infectious causes of DAD were not found at post mortem in any of these cases. The extent of ante mortem clinical evaluation to exclude infection was variable, 12 patients were found to have had cultures in their respiratory secretions. In addition, cultures of bronchoalveolar fluid were performed in four patients and surgical lung biopsy specimens in three patients. Viral cultures were performed in only four patients (two surgical lung biopsy, two bronchoalveolar fluid and one sputum) and did not yield a pathogen. The remaining five patients (without DAD) died from the gradual progression of IPF resulting in respiratory failure (table 2).

A total of $10(24 \%)$ patients died from respiratory causes other than IPF including pneumonia, aspiration and drug-induced lung disease. In the one patient with drug-induced lung disease diagnosis included the histopathological demonstration of DAD in the setting of chemotherapy (cyclophosphamide, doxorubicin and vincristine), used in the treatment of lymphoma and not acute exacerbation of IPF. Those dying of pneumonia included five patients with ventilator-associated pneumonia caused by nosocomial pathogens, two patients with community-acquired pneumonia (CAP) and one patient with blastomycosis.

\section{TABLE 2 Immediate cause of death}

Causes

Cases n (\%)

$\begin{array}{ll}\text { Respiratory } & 27(64) \\ \text { Acute exacerbation of IPF } & 12(29) \\ \text { Gradual progression of IPF } & 5(12) \\ \text { Pneumonia } & 8(15) \\ \text { Aspiration } & 1(2) \\ \text { Drug-induced lung disease } & 1(2) \\ \text { Cardiovascular } & 9(21) \\ \text { Arrhythmia } & 3(7) \\ \text { Myocardial infarction } & 3(7) \\ \text { Cor pulmonale } & 2(3) \\ \text { Stroke } & 1(3) \\ \text { Other } & 6(14) \\ \text { Multiorgan failure } & 2(5) \\ \text { Trauma } & 2(5) \\ \text { Acute renal failure } & 1(3) \\ \text { Anoxic encephalopathy } & 1(3)\end{array}$

IPF: idiopathic pulmonary fibrosis. 


\section{Cardiovascular causes}

Cardiovascular disease was the cause of death in nine $(21 \%)$ subjects. These included three $(7 \%)$ patients with sudden death in whom the most likely cause of death was arrhythmia. Myocardial infarction, severe cor pulmonale and a stroke accounted for the remaining cardiovascular causes (table 2).

Pulmonary hypertension or cor pulmonale was the immediate cause of death in two patients. However, 17 (40\%) of the 42 subjects that had undergone a post mortem showed post mortem evidence for pulmonary hypertension or cor pulmonale as contributing factors for mortality (table 3). Although only four patients had a clinical diagnosis of pulmonary hypertension recorded in their medical records prior to a post mortem, echocardiography had been performed within 2 months prior to death in $23(55 \%)$ patients, $13(57 \%)$ of whom had echocardiographical evidence of right ventricular enlargement and/or dysfunction. Pulmonary artery systolic pressure was assessable in 21 patients; median (range) estimated pulmonary artery systolic pressure was $55(30-105) \mathrm{mmHg}$. Estimated pulmonary artery systolic pressure was elevated $(>30 \mathrm{mmHg})$ in all but one of these patients. Of the 17 patients who had post mortem evidence of pulmonary hypertension or cor pulmonale, 12 had undergone echocardiography. Pulmonary hypertension was demonstrated by echocardiography in 11 (92\%) of these patients. No patient was known to be receiving treatment specifically for pulmonary hypertension. Three patients who died as a result of dysrhythmia, without direct evidence of coronary artery disease, had post mortem evidence for moderate-to-severe pulmonary hypertension as a contributing factor. From the 17 patients, in whom IPF was the immediate cause of death, eight (47\%) had post mortem evidence for pulmonary hypertension or cor pulmonale.

\section{Noncardiopulmonary causes}

The causes of noncardiopulmonary deaths included trauma, multiorgan failure, anoxic encephalopathy (post-operative upper airway obstruction) and renal failure. The two cases of traumatic death were one of a motor-vehicle accident and fallrelated injuries.

\section{Infections}

All 31 hospitalised patients had respiratory cultures and 27 (87\%) had blood cultures. A total of $15(48 \%)$ patients had cultures obtained through interventional techniques; bronchoalveolar lavage $(n=9)$ and open lung biopsy $(n=6)$. Overall culture yield was low with pathogenic growth evident in nine (29\%) patients. Only four of the nine subjects had culture evidence for respiratory infection at presentation with five of the nine developing ventilator-associated pneumonia during prolonged hospital stay with mechanical ventilation. In one patient blastomycosis was not diagnosed ante mortem. CAP was diagnosed from cultures found in the respiratory secretions of two patients; one patient had Haemophilus influenzae and one had H. influenzae and Streptococcus pneumoniae. Another patient had aspiration pneumonia and culture evidence for Enterobacter aerogenes. Ventilator-associated pneumonia was diagnosed in five patients with hospitalacquired organisms including Stenotrophomonas maltophilia, Pseudomonas aeruginosa, Enterobacter sp. and methicillin-resistant Staphylococcus aureus.

\section{Other findings}

The diagnosis of IPF was not known at the time of death in nine $(21 \%)$ patients and included four patients who presented with acute exacerbation of IPF, two patients with trauma, one patient with multiorgan failure, one with severe tracheobronchitis, and one patient with gradual progression of IPF, which was not recognised ante mortem. The immediate cause of death was clinically unsuspected in five $(12 \%)$ patients and included three patients with pneumonia, one with severe cor pulmonale and one with cardiac disease.

\section{DISCUSSION}

The causes of death in patients with IPF have not been clearly defined and relatively few post mortem studies have been performed. In the current study the immediate cause of death was found to be respiratory related in the majority of patients with acute exacerbation of IPF being the single most common cause.

In 1990, PANOs et al. [9] summarised six studies published between 1964 and 1983 and found respiratory failure to be the most common cause of death (39\%) among 326 patients with IPF. Other causes of death included heart failure (14\%), bronchogenic carcinoma $(10 \%)$, ischaemic heart disease $(10 \%)$, nonpulmonary infection $(4 \%)$, pulmonary embolism $(3 \%)$, stroke $(3 \%)$ and pulmonary infection (3\%).

MARTINEZ et al. [3] recently described the clinical course of 168 patients with mild-to-moderate IPF in the placebo group of a randomised, controlled clinical trial. During the observation period (median 76 weeks) 21\% of the subjects died. Death was

\section{TABLE 3 Contributing causes of death}

\begin{tabular}{|c|c|}
\hline Causes & Cases n (\%) \\
\hline Respiratory Conditions & $31(74)$ \\
\hline IPF & $25(60)$ \\
\hline Emphysema & $5(12)$ \\
\hline Pneumonia & $4(10)$ \\
\hline Pulmonary embolism & $1(2)$ \\
\hline Bronchiectasis & $1(2)$ \\
\hline Radiation fibrosis & $1(2)$ \\
\hline Cardiovascular & $24(57)$ \\
\hline Cor pulmonale & $17(40)$ \\
\hline Ischaemic heart disease & $13(31)$ \\
\hline Cerebrovascular disease & $3(7)$ \\
\hline Other & $24(57)$ \\
\hline Chronic renal disease & $9(21)$ \\
\hline Chronic liver disease & $4(10)$ \\
\hline Cancer & $4(10)$ \\
\hline Dementia & $3(7)$ \\
\hline Surgical complications & $3(7)$ \\
\hline Sepsis & $2(5)$ \\
\hline Hypoxic encephalopathy & $2(5)$ \\
\hline Gastroduodenal ulcer & $2(5)$ \\
\hline Acute pancreatitis & $1(2)$ \\
\hline Radiation therapy & $1(2)$ \\
\hline
\end{tabular}

Data are presented as $n(\%)$. IPF: idiopathic pulmonary fibrosis. 
considered to be IPF-related in $89 \%$ of these patients; progression of IPF was the primary cause of death in $56 \%$ of the patients. Death was categorised as acute or abrupt in approximately half of the patients with an IPF-related death; primary cause of death in these patients included progression of IPF (17\%, of all deaths), pneumonia (11\%), acute respiratory distress syndrome $(6 \%)$, cor pulmonale $(3 \%)$, and unknown $(6 \%)$. Most of the remaining patients died from subacute progression of IPF (39\% of all deaths). The cause of death in the study by MARTINEZ et al. [3] was assigned by individual clinicians responsible for the research participants. How many of these patients underwent a post mortem was not stated.

ARAKI et al. [10] reported a consecutive post mortem series of 86 elderly patients (aged $>65$ yrs) in Japan with IPF in whom the most common causes of death included: bacterial pneumonia (23\%), progressive IPF $(20 \%)$, lung cancer $(17.4 \%)$ or an acute exacerbation of IPF $(10.5 \%)$. The mean age of the patients studied by ARAKI et al [10] was 80.5 yrs and $66 \%$ of the patients were reported as current or previous smokers.

RUDD et al. [11] recently reported the results of a British Thoracic Society survey of patients with IPF (or cryptogenic fibrosing alveolitis). Among the 398 deaths reported, the different causes of death included were: primarily due to IPF (54\%); partly due to IP (18\%); due to lung cancer with IPF or other cause $(12 \%)$; and other causes $(16 \%)$. These data were derived from death certificates; the number of patients who had undergone a post mortem was not defined. A similar study in the USA determined that $60 \%$ of patients with IPF died as a direct result of their lung disease [12].

The current study's post mortem observations provide a unique perspective on the causes of death in patients with IPF. In all of the present study's patients, IPF was either the immediate cause $(40 \%)$ or a contributing cause $(60 \%)$ of death. Acute exacerbation of IPF is a phenomenon that has only gained attention in recent years. In contrast to previous studies [3, 10], the present results suggest acute exacerbation of IPF to be the most common cause of death in these patients as manifested by DAD superimposed on UIP without any precipitating cause other than the underlying lung disease. However, the present authors acknowledge that present findings may have been biased by the increased probability of post mortems being undertaken on patients who die from an acute clinical worsening with no clear explanation compared with the number of post mortems being performed on patients with expected gradual progression of IPF.

Diffuse alveolar damage superimposed on UIP was present in all patients who met clinical criteria for acute exacerbation of IPF [7]. However, four other patients with varied presentations also had histopathological evidence for DAD; three of these patients had identifiable causes for DAD that included aspiration, CAP and chemotherapy-induced acute lung injury. Arrhythmia was the immediate cause of death in the remaining patient. In patients with DAD who met criteria for acute exacerbation of IPF, infectious aetiologies were not identified ante or post mortem. These findings suggest the aetiology of acute exacerbation of IPF is either noninfectious or not easily detected by conventional pathology and laboratory techniques.
Pulmonary hypertension has recently been identified as a relatively common risk factor for shortened survival in patients with IPF [13-15]. Evidence of pulmonary hypertension or cor pulmonale was present in almost half of the patients in the current study. The present authors' results are similar to those of LETTIERI et al. [14] who found pulmonary arterial hypertension to be present in $32 \%$ of the 79 patients undergoing pretransplantation right heart catheterisation.

The current study identified nine $(21 \%)$ patients who did not have IPF diagnosed ante mortem. In five of these undiagnosed cases, IPF was the immediate cause of death and in four of the subjects the initial presentation of IPF was acute exacerbation of IPF with DAD on UIP at post mortem. This finding confirms prior reports that suggested acute exacerbation of IPF may be the initial presentation in some patients with IPF who have minimal symptoms and have not previously presented for a medical evaluation [4]. In addition, prevalence reports of IPF which rely on clinician's death-certificate diagnoses may underestimate the true prevalence of IPF.

Aside from the bias associated with a post mortem examination as already discussed, there are other limitations to the present study. The current study includes a relatively modest number of patients and represents a small fraction of the total number of patients seen at the Mayo Clinic with a diagnosis of IPF. Not all patients with IPF who died at the Mayo Clinic underwent a post mortem examination. The post mortem series may be enriched for atypical cases since the Mayo Clinic medical centre is a tertiary referral institution. Clinical selection of diagnostically challenging cases may have skewed the data although clinicians appear to have little ability in identifying cases that might yield diagnostic surprises [16-19]. Comfortcare services would be appropriate for patients dying of progressive respiratory failure in the setting of advanced pulmonary fibrosis and these patients may be under represented in a post mortem study. Similarly, patients with progressive lung cancer are more likely to have been diagnosed before death and are also more probable to be under represented in this post mortem series. It is also acknowledged that there are difficulties in identifying the precise cause of death in elderly patients in whom comorbidities are prevalent. The present authors acknowledge the current findings may over represent difficult or unusual IPF cases due to the artificial selection of such cases for post mortem. Thereby the extrapolation of the present results to IPF patients in general requires clinical judgment.

To summarise, the current authors conclude that the majority of patients with idiopathic pulmonary fibrosis undergoing a post mortem examination had experienced a respiratory-related death and that an acute exacerbation of the underlying disorder is the most common immediate cause.

\section{REFERENCES}

1 Bjoraker JA, Ryu JH, Edwin MK, et al. Prognostic significance of histopathologic subsets in idiopathic pulmonary fibrosis. Am J Respir Crit Care Med 1998; 157: 199-203.

2 Daniels CE, Ryu JH. Treatment of idiopathic pulmonary fibrosis. Semin Respir Crit Care Med 2006; 27: 668-676. 
3 Martinez FJ, Safrin S, Weycker D, et al. The clinical course of patients with idiopathic pulmonary fibrosis. Ann Intern Med 2005; 142: 963-967.

4 Parambil JG, Myers JL, Ryu JH. Histopathologic features and outcome of patients with acute exacerbation of idiopathic pulmonary fibrosis undergoing surgical lung biopsy. Chest 2005; 128: 3310-3315.

5 Hutchins GM, Berman JJ, Moore GW, Hanzlick R. Practice guidelines for autopsy pathology: autopsy reporting. Autopsy Committee of the College of American Pathologists. Arch Pathol Lab Med 1999; 123: 1085-1092.

6 American Thoracic Society. Idiopathic pulmonary fibrosis: diagnosis and treatment. International consensus statement. American Thoracic Society (ATS), and the European Respiratory Society (ERS). Am J Respir Crit Care Med 2000; 161: 646-664.

7 Kondoh Y, Taniguchi H, Kawabata Y, Yokoi T, Suzuki K, Takagi K. Acute exacerbation in idiopathic pulmonary fibrosis. Analysis of clinical and pathologic findings in three cases. Chest 1993; 103: 1808-1812.

8 Akira M, Hamada H, Sakatani M, Kobayashi C, Nishioka M, Yamamoto S. CT findings during phase of accelerated deterioration in patients with idiopathic pulmonary fibrosis. AJR Am J Roentgenol 1997; 168: 79-83.

9 Panos RJ, Mortenson RL, Niccoli SA, King TE Jr. Clinical deterioration in patients with idiopathic pulmonary fibrosis: causes and assessment. Am J Med 1990; 88: 396-404.

10 Araki T, Katsura H, Sawabe M, Kida K. A clinical study of idiopathic pulmonary fibrosis based on autopsy studies in elderly patients. Intern Med 2003; 42: 483-489.
11 Rudd RM, Prescott RJ, Chalmers JC, Johnston ID. British Thoracic Society Study on cryptogenic fibrosing alveolitis: response to treatment and survival. Thorax 2007; 62: 62-66.

12 Olson AL, Swigris JJ, Lezotte DC, Norris JM, Wilson CG, Brown KK. Mortality from pulmonary fibrosis increased in the United States from 1992 to 2003. Am J Respir Crit Care Med 2007; 176: 277-284.

13 Nadrous HF, Pellikka PA, Krowka MJ, et al. The impact of pulmonary hypertension on survival in patients with idiopathic pulmonary fibrosis. Chest 2005; 128: Suppl. 6, 616S-617S.

14 Lettieri CJ, Nathan SD, Barnett SD, Ahmad S, Shorr AF. Prevalence and outcomes of pulmonary arterial hypertension in advanced idiopathic pulmonary fibrosis. Chest 2006; 129: 746-752.

15 Nathan SD, Noble PW, Tuder RM. Idiopathic pulmonary fibrosis and pulmonary hypertension: connecting the dots. Am J Respir Crit Care Med 2007; 175: 875-880.

16 Britton M. Diagnostic errors discovered at autopsy. Acta Med Scand 1974; 196: 203-210.

17 Cameron HM, McGoogan E. A prospective study of 1152 hospital autopsies: I. Inaccuracies in death certification. J Pathol 1981; 133: 273-283.

18 Landefeld CS, Chren MM, Myers A, Geller R, Robbins S, Goldman L. Diagnostic yield of the autopsy in a university hospital and a community hospital. N Engl J Med 1988; 318: 1249-1254.

19 Shojania KG, Burton EC, McDonald KM, Goldman L. Changes in rates of autopsy-detected diagnostic errors over time: a systematic review. Jama 2003; 289: 2849-2856. 